Table des matières du tome LXXXX, fascicule 1

P. G. Hinman, The finite levels of the hierarchy of effective $R$-sets . . . $\quad$ Pages B. Leclerc et B. Monjardet, Ordres "C.A.C." . . . . . . . . . . . . 11-22 S. E. Rodabaugh, Retracts of Tychonoff and normal spaces. . . . . . 23-31 J. Schmid, on the compactification of closure algebras . . . . . . . . 33-48 K. C. Millett, Homotopy sequences of fibrations . . . . . . . . . 49-62 R. J. Daverman, On the scarcity of tame disks in certain wild cells . . . . 63-77 U. Felgner and T. J. Jech, Variants of the axiom of choice in set theory

with atoms. . . . . . . . . . . . . . 79-85

Les FUNDAMENTA MATHEMATICAE publient, en langues des congrès internationaux, des travaux consacrés à la Théorie des Ensembles, Topologie, Fondements de Mathématiques, Fonctions Réelles, Algèbre Abstraite Chaque volume paraît en 3 fascicules

Adresse de la Rédaction et de l'Échange:

FUNDAMENTA MATHEMATICAE, Sniadeckich 8, 00-950 Warszawa 1 (Pologne)

Tous les volumes sont à obtenir par l'intermédiaire de

ARS POLONA-RUCH, Krakowskie Przedmieście 7, 00-068 Warszawa 1 (Pologne)

\section{The finite levels of the hierarchy of effective $R$-sets}

by

Peter G. Hinman (Ann Arbor, Michigan)

Abstract. Let $R_{0}$ be the operation of countable intersection, $R_{n+1}$ the operation obtained by applying Kolmogorov's operator $R$ to the dual of $R_{n}, R_{n}$ the class of subsets of $\omega$ obtained by applying $R_{n}$ to recursive families of subsets of $\omega$, and $e R_{n}$ the class of complements of sets in $R_{n}$. Then $R_{0}=\Pi_{1}^{0}, R_{1}=\Sigma_{1}^{1}$, and for all $n, R_{n} \subseteq \Delta_{2}^{1}$. We are concerned here with the question of which of the many methods which in some sense "generate" $\Delta_{1}^{1}$ from $\Pi_{1}^{0}$ also suffice to "generate" $\mathcal{R}_{n+1} \cap a \Re_{n+1}$ from $\mathcal{R}_{n}$. First we show that the iteration of a jump operator $j$ over a system of ordinal notations does not suffice: if $j$ is the hyperjump or indeed any jump definable in $\mathcal{R}_{2} \cap \circ \mathcal{R}_{2}$ form, the resulting class of sets is properly included in $\mathcal{R}_{2} \cap e \mathcal{R}_{2}$. On the other hand we define a set $K_{n+1}$ and a prewell-ordering on it such that the classes of sets reducible to segments of $K_{n+1}$ form a hierarchy for $\mathcal{R}_{n+1} \cap \propto \mathcal{R}_{n+1}$. Finally we show that $A \in C \mathcal{R}_{n+1}$ just in case it is reducible to a set inductively defined by a monotone operator in $\mathbb{R}_{n}$ form.

Several recent articles ([Am 1, 2], [Hi 1]) have considered various aspects of the interplay between Kolmogorov's operator $\boldsymbol{R}$ and hierarchies of recursion theory. In this paper we contribute to this development and in particular answer a question posed in [Am 1] concerning the relative extent of several hierarchies. Our notation will follows that of [Am 1] and [Hi 1] as much as possible and the reader will profit from some familiarity with these papers. The set of natural numbers is denoted by $N$ and the set of number-theoretic functions by $N^{N}$. Variables $a, b, c, \ldots$ range over $N ; \alpha, \beta, \gamma, \ldots$ over $N^{N}$; and $\varphi, \psi, \theta, \ldots$ over the set of partial functions from some $N^{k}$ into $N$. We identify finite sequences of natural numbers with their images under some fixed encoding and use letters $s, t, u$ to denote them; $s\langle m\rangle$ is a sequence of length one greater than that of $s, s * t$ is the concatenation of $s$ and $t$, and $s \subseteq t$ means $s$ is an initial segment of $t$. Dm and $\mathrm{Rg}$ denote domain and range respectively.

1. The $R$-hierarchies. If $T$ is an operation on countable families of subsets of $N$, we say $\Gamma$ has base $M \subseteq N^{N}$ iff for any family $F$ and any $m \in N$

$$
m \in \Gamma(F) \leftrightarrow \leftrightarrow \alpha_{a \in M} \nabla p[m \in F(\alpha(p))] .
$$

1-Fundamenta Mathematicae, T. IXXIX 
We shall need to consider the following bases closely connected with $M$ :

$$
\begin{aligned}
M^{0}= & \left\{\gamma \mid \forall \alpha_{a \in M} \mathbb{H} p[\alpha(p) \in \operatorname{Rg} \gamma]\right\}, \\
M^{*}= & \left\{\gamma \mid \mathbb{H} \alpha_{a \in M} \forall p \forall \beta_{\beta \in M} \mathbb{H} q[\langle\alpha(p), \beta(q)\rangle \in \operatorname{Rg} \gamma]\right\}, \\
R M= & \{\gamma \mid \forall t \forall u \\
& \text { (i) } \varnothing \in \operatorname{Rg} \gamma, \\
& \text { (ii) } u \subseteq t \in \operatorname{Rg} \gamma \rightarrow u \in \operatorname{Rg} \gamma, \text { and } \\
& \text { (iii) } \left.t \in \operatorname{Rg} \gamma \rightarrow \mathbb{H} \alpha_{a \in M} \forall p[t\langle\alpha(p)\rangle \in \operatorname{Rg} \gamma]\right\} .
\end{aligned}
$$

Although an operation $\Gamma$ will in general have many bases, it is obvious that $M^{0}, M^{*}$, and $\boldsymbol{R} M$ depend only on $\Gamma$ so we may write $\Gamma^{0}, \Gamma^{*}$, and $\boldsymbol{R} \Gamma$ for the operations with these bases.

We wish first to consider the two sequences of operations:

$$
\begin{aligned}
& Q_{0}=\bigcap \text { (countable intersection), } \quad Q_{n+1}=\boldsymbol{R}\left(Q_{n}^{*}\right), \\
& R_{0}=\bigcap, \quad R_{n+1}=\boldsymbol{R}\left(R_{n}^{0}\right)
\end{aligned}
$$

Each operator generates a class of sets of natural numbers:

$$
\begin{aligned}
& Q_{n}=\left\{Q_{n}(F) \mid F \text { is a recursive family }\right\}, \\
& \mathcal{R}_{n}=\left\{R_{n}\left(F^{\prime}\right) \mid F \text { is a recursive family }\right\},
\end{aligned}
$$

where $F$ is a recursive family iff the relation $m \in F(p)$ is recursive. The classes $R_{0}$ and $R_{1}$ are well-understood, being respectively $\Pi_{1}^{0}$ and $\Sigma_{1}^{1}$. We denote by $c Q_{n}$ and $c R_{n}$ the class of complements of $Q_{n}$ and $\mathcal{R}_{n}$ sets.

Let $L_{n}$ and $M_{n}$ be the natural bases for the operations $Q_{n}$ and $R_{n}$ respectively - that is, $L_{0}=M_{0}=\{\lambda p \cdot p\}, L_{n+1}=\boldsymbol{R} L_{n}^{*}$, and $M_{n+1}=\boldsymbol{R} M_{n}^{0}$. In accord with [Hi 1, 5.1], we define functionals $q_{n}^{*}$ and $r_{n}^{*}$ as follows: for any (partial) function $\varphi$,

$$
\begin{aligned}
& q_{n}^{*}(\varphi)\left\{\begin{array}{l}
\simeq 0, \quad \text { if } \quad \mathbb{H} \alpha_{a \in L_{n}} \nabla p[\varphi(\alpha(p)) \simeq 0], \\
\simeq 1, \quad \text { if } \quad \forall \alpha_{a \in L_{n}} \mathbb{H} p[\varphi(\alpha(p)) \simeq 1], \\
\text { is undefined, otherwise; }
\end{array}\right. \\
& r_{n}^{*}(\varphi)\left\{\begin{array}{l}
\simeq 0, \text { if } \mathbb{H} \alpha_{a \in M_{n}} \nabla p[\varphi(\alpha(p)) \simeq 0], \\
\simeq 1, \text { if } \forall \alpha_{a \in M_{n}} \mathbb{H} p[\varphi(\alpha(p)) \simeq 1], \\
\text { is undefined, otherwise. }
\end{array}\right.
\end{aligned}
$$

From [Hi 1], 7.5, we have for all $A \subseteq N$ and all $n$ :

$$
\begin{gathered}
A \in c Q_{n+1} \leftrightarrow A \quad \text { is recursively enumerable in } q_{n}^{*}, \\
A \in Q_{n+1} \cap c Q_{n+1} \leftrightarrow A \text { is recursive in } q_{n}^{\#} .
\end{gathered}
$$

Our first goal is to establish the corresponding result for the $\mathcal{R}_{n}$ and $r_{n}^{*}$. In fact, we shall see that the operations $Q_{n}$ and $R_{n}$ are equivalent, the classes $Q_{n}$ and $R_{n}$ coincide, and the functionals $q_{n}^{*}$ and $r_{n}^{\#}$ are recursive in each other. We recall from [Hi 1], 2.9, that for any two operations $I$ and $\Lambda, \Gamma$ subsumes $\Lambda(\Gamma \geqslant \Lambda)$ iff there exists a primitive recursive function $\pi$ such that for any family $F$,

$$
\Lambda(F)=\Gamma(\lambda p \cdot F(\pi(p)))
$$

Note that is a transitive relation. We will say that $\Gamma$ and $A$ are equivalent $(\Gamma \sim \Lambda)$ iff both $\Gamma \geqslant \Lambda$ and $\Lambda \geqslant \Gamma$.
LEMMA. For any operations $\Gamma$ and $A$ :
(a) $\Gamma^{00}=\Gamma$,
(b) $\Gamma \geqslant \Lambda \rightarrow I^{0} \geqslant \Lambda^{0}$,
(c) $\Lambda^{*} \geqslant \Lambda$ and $\Lambda^{*} \geqslant \Lambda^{0}$,
(d) $R \Gamma \geqslant \Gamma$
(e) $\Gamma \geqslant \Lambda \rightarrow R \Gamma \geqslant \boldsymbol{R} \Lambda$,
(f) $R \Gamma \geqslant \Lambda$ and $R \Gamma \geqslant \Lambda^{0} \rightarrow R \Gamma \geqslant \Lambda^{*}$,
(g) $R \Gamma \sim R R \Gamma$.

Proof. (a) and (b) are trivial, (c) is proved just before [Hi 1], 4.7, (d) and (e) are [Hi 1], 4.6, (f) follows easily from [Hi 1], 4.7, and (g) follows from (d) and the proof of [Hi 1], 4.7.

THeOREM 1. For all $n, Q_{n} \sim R_{n}$.

Proof. For $n=0$ this is obvious so assume $Q_{n} \sim R_{n}$ as induction hypothesis. From $Q_{n} \geqslant R_{n}$ we derive successively $Q_{n}^{0} \geqslant R_{n}^{0}$ by (b) of the lemma, $Q_{n}^{*} \geqslant R_{n}^{0}$ by (c) and transitivity, and $Q_{n+1} \geqslant R_{n+1}$ by (e). Toward the converse, we have first $R_{n+1} \geqslant R_{n}^{0} \geqslant Q_{n}^{0}$ by (d), (b), and the induction hypothesis. If $n=0, R_{n+1} \geqslant Q_{n}$ is well-known [Hi 1], 2.9, examples. Otherwise, $R_{n} \geqslant Q_{n}=\boldsymbol{R} Q_{n-1}^{*} \geqslant Q_{n-1}^{*} \geqslant Q_{n-1}, Q_{n-1}^{0}$ by the induction hypothesis, (d), and (c). Hence $R_{n+1} \geqslant R_{n}^{0} \geqslant Q_{n-1}^{0}, Q_{n-1}$ by (d) and (b), so $R_{n+1} \geqslant Q_{n-1}^{*}$ by (f). Hence $R_{n+1}^{n} \sim \boldsymbol{R} R_{n+1} \geqslant \boldsymbol{R} Q_{n-1}^{*}=Q_{n}$ by (g) and (e). Finally, $R_{n+1} \geqslant Q_{n}^{*}$ by (f) and again applying (g) and (e) we obtain $R_{n+1}$ $\geqslant R R_{n+1} \geqslant R Q_{n}^{*}=Q_{n+1}$ as required.

COROLLARY A. For all $n, Q_{n}=\mathcal{R}_{n}$ and $q_{n}^{*}$ and $r_{n}^{*}$ are recursive in each other.

COROLLARY B. For all $A \subseteq N$ and all $n$ :

(a) $A \in c \mathbb{R}_{n+1} \leftrightarrow A$ is recursively enumerable in $r_{n}^{\#}$,

(b) $A \in \mathbb{R}_{n+1} \cap e \mathcal{R}_{n+1} \leftrightarrow A$ is recursive in $r_{n}^{*}$.

The functional $r_{1}^{*}$ has been studied by Aczel in [Ac] where it is called $E_{1}^{*}$. Since $R_{1}$ is the operation $\mathcal{A}$ it is easy to see that for any $\varphi$ :

$$
E_{1}^{*}(\varphi)\left\{\begin{array}{l}
\simeq 0, \text { if } \quad \mathbb{H} \alpha \nabla n \mid \varphi(\bar{\alpha}(n)) \simeq 0], \\
\simeq 1, \text { if } \quad \forall a \mathbb{H} n[\varphi(\bar{\alpha}(n)) \simeq 1], \\
\text { is undefined, otherwise. }
\end{array}\right.
$$

Then we have immediately 
CoRollary C. For all $A \subseteq N$ :

(a) $A \in c \mathcal{R}_{2} \leftrightarrow A$ is recursively enumerable in $E_{1}^{*}$,

(b) $A \in \mathcal{R}_{2} \cap 0 \mathcal{R}_{2} \leftrightarrow A$ is recursive in $E_{1}^{\#}$ :

2. Short hierarchies. In [Am 1], 33 , Amstislavskii considers severa] hierarchies of subsets of $N$ and shows that all of the sets involved lie in $\mathcal{R}_{2} \cap c \mathcal{R}_{2}$ but leaves open the question whether or not any of these hierarchies exhaust $\mathcal{R}_{2} \cap c \mathcal{R}_{2}$. In this section we shall show that these hierarchies comprise only part of $\mathcal{R}_{2} \cap c \mathcal{R}_{2}$ and indeed, in some sense, only a very small part.

Let $E_{1}$ denote the restriction of $E_{1}^{*}$ to total functions. In view of Corollary $\mathrm{C}$ and the following immediate consequence of [Hi 1], 5.11:

(7) The class of sets $A \subseteq N$ recursive in $E_{1}$ is properly included in the class of sets recursive in $E_{1}^{*}$,

we need only show that all of the sets in the hierarchies of [Am 1], $\S 3$ are recursive in $E_{1}$.

In accord with the notation of [Am 1], $\S 3$, let $<_{w}$ be a well-founded partial ordering of a set $W \subset N$ with least element 1 . If $j: \mathfrak{T}(N) \rightarrow \mathfrak{T}(N)$, then $\pi\left(j,<_{w}\right)$ is the class of those functions $\mathscr{F}: W \rightarrow \mathcal{S}(N)$ such that there exist partial recursive $\varphi, \chi$, and $\psi$ such that for all $a, b, c$ with $a \in W \sim\{1\}$ :

(i) $\varphi(a)<{ }_{w} a$,

(ii) $\chi(a, b) \in \mathscr{F}(\varphi(a)) \rightarrow b<{ }_{w} a$, and

(iii) $c \in \mathscr{F}(a) \leftrightarrow \psi(a, c) \in j\left(\nabla_{a}\right)$, where

$$
V_{a}=\{\langle b, c\rangle \mid \chi(a, b) \in \mathscr{F}(\varphi(a)) \text { and } c \in \mathscr{F}(b)\} .
$$

We write $A=[e]$ to mean that $\lambda m \cdot\{e\}\left(E_{1}, m\right)$ is the characteristic function of $A$. If $h j$ denotes the hyperjump, a basic property of $E_{1}$ is that there exists a primitive recursive $\tau$ such that $h j([e])=[\tau(e)]$.

THEOREM 2. If $\mathcal{F}_{\epsilon} \in\left(h j,<_{w}\right)$ and $\mathcal{F}(1)$ is recursive in $E_{1}$, then there exists a partial recursive function $\varrho$ such that for all $a \in W, \mathscr{F}(a)=[\varrho(a)]$. In particular, each $F(a)$ is recursive in $E_{1}$.

Proof. Following closely the proof of [Am 1], Theorem 3.1, we assume for application of the recursion lemma that $a \in W$ and

Let

$$
\left(\nabla b<_{w} a\right)(\mathcal{F}(b)=[\{l\}(b)]) .
$$

$$
\begin{aligned}
U & =\{\langle l, a, b, c\rangle \mid \chi(a, b) \in[\{l\}(\varphi(a))] \text { and } c \in[\{l\}(b)]\} \\
& =\left\{\langle l, a, b, c\rangle \mid\{\{l\}(\varphi(a))\}\left(E_{1}, \chi(a, b)\right) \simeq 0 \text { and }\{\{l\}(b)\}\left(E_{1}, c\right) \simeq 0\right\} .
\end{aligned}
$$

$U$ is only recursively enumerable in $E_{1}$, but for $a \in W, V_{a}=\{\langle b, c\rangle \mid$ $\langle l, a, b, c\rangle \in U\}$ is recursive in $E_{1}$. In fact, there exists a primitive re- cursive $\sigma$ such that $\nabla_{a}=[\sigma(l, a)]$. Hence $h j\left(\nabla_{a}\right)=[\tau(\sigma(l, a))]$ and given an index for $\psi$ it is easy to compute an $E_{1}$-index for $\mathscr{F}(a)$.

Now if $H_{h}(a)$ and $\mathfrak{h}_{a}$ are the sets obtained by iterating the hyperjump over, respectively, the notation systems $D_{h}$ of [En] and $\widetilde{C}$ of [Kr-Ro], we have

COROLLARY D. For all $a \in D_{h}, H_{h}(a)$ is recursive in $E_{1}$. For all $a \in \widetilde{C}$, $\mathfrak{h}_{a}$ is recursive in $E_{1}$. Hence the class of sets recursive in some $H_{h}(a)$ or $\mathfrak{h}_{a}$ is properly included in $\boldsymbol{R}_{2} \cap a \Re_{2}$.

Proof. For $H_{h}$ this is an immediate consequence of Theorem 2, [Am 1], Lemma 3.2, and (7). The methods used to establish the results for $h_{a}$ are similar to those used in the proof of Theorem 2 .

Theorem 2 can be considerably strengthened. The following im provement of (7) is an immediate consequence of [Ac], Theorem 3:

(8) If $f$ is a functional with domain $N^{N}$ and recursive in $E_{1}^{\#}$, then the class of sets $A \subseteq N$ recursive in $f$ is properly included in the class of sets recursive in $E_{1}^{\#}$.

Let us eall $j$ an $\mathcal{R}_{2} \cap e \mathcal{R}_{2} j u m p$ operator iff there exist recursive relations $S$ and $T$ such that for any $A \subseteq N$ :

$$
\begin{aligned}
j(A) & =\left\{m \mid \mathbb{H} \alpha_{a \in M_{2}} \nabla p S(A, m, \alpha(p))\right\} \\
& =\left\{m \mid \nabla \alpha_{\alpha \in M_{2}} \mathbb{H} p T(A, m, \alpha(p))\right\} .
\end{aligned}
$$

Let $f_{j}$ be the functional defined for total $\alpha$ by:

$$
f_{j}(\alpha, m)= \begin{cases}0, & \text { if } \quad m \in j(\{n \mid \alpha(n)=0\}) \\ 1, & \text { otherwise }\end{cases}
$$

From the extended version of [Hi 1], 7.5 established in [Hi 1], § 8, we have (9) for any $\mathcal{R}_{2} \cap e \mathcal{R}_{2}$ jump operator $j, f_{j}$ is recursive in $E_{1}^{\#}$.

TheOREM 3. If $j$ is any $\mathfrak{R}_{2} \cap c \mathfrak{R}_{2}$ jump operator, $\mathscr{F} \in \pi\left(j,<_{w}\right)$, and $\mathscr{F}(1) \in \mathcal{R}_{2} \cap c \mathcal{R}_{2}$, then the class of sets recursive in some $\mathscr{F}(a)$ with $a \in W$ is properly included in $\mathfrak{R}_{2} \cap e R_{2}$.

Proof. By (9) and Corollary $C$ there exists a functional $g$ defined only on total arguments, recursive in $E_{1}^{*}$, and such that $f_{j}$ and $\mathscr{F}(1)$ are both recursive in $g$. As in the proof of Theorem 2 we may construct a recursive function $\varrho$ such that for all $a \in W, \varrho(a)$ is a $g$-index for $F(a)$. Hence the class of sets recursive in some $\mathscr{F}(a)$ with $a \in W$ is included in the class of sets recursive in $g$, which is properly included in $\mathcal{R}_{2} \cap c \mathcal{R}_{2}$ by (8) and Corollary $\mathrm{C}$.

A similar result holds for all $\mathcal{R}_{n} \cap e \mathcal{R}_{n}, n \geqslant 2$. 
3. Longer hierarchies. Although the above hierarchies do not exhaust $\mathcal{R}_{n+1} \cap c \mathcal{R}_{n+1}$, we shall construct one here that does. In [Hi 1$]$, 5.2 we defined for each operation $\Gamma$ a set $K^{T}$ and a mapping ||$^{T}$ of $K^{T}$ onto an ordinal $\varkappa^{\Gamma}$. Let $K_{n},|| n$, and $\varkappa_{n}$ be the set, mapping, and ordinal corresponding to $\Gamma=R_{n}$. The following facts are easily derived from [Hi 1], $5.4,7.3,7.4$, and 5.7:

(10) $A$ is recursively enumerable in $r_{n}^{*} \leftrightarrow A$ is many-one reducible to $K_{n}$.

(11) There exists a function $\eta$ partial recursive in $r_{n}^{\#}$ such that (i) $u \in K_{n}$ and $|u|_{n} \leqslant|v|_{n \rightarrow \eta} \rightarrow(u, v) \simeq 0$, and (ii) $|v|_{n}<|u|_{n \rightarrow \eta}(u, v) \simeq 1$.

For each $v<x_{n}$, let $K_{n}(\nu)=\left\{u \mid u \epsilon K_{n}\right.$ and $\left.|u|_{n} \leqslant \nu\right\}$. Then from (11) we have immediately

$$
v<\varkappa_{n} \rightarrow K_{n}(v) \text { is recursive in } r_{n}^{\#} \text {. }
$$

If we combine this with (10) and the boundedness argument of [Hi 1], 5.9, we obtain for any $A \subseteq N$ :

(13) $A$ is recursive in $r_{n}^{*} \leftrightarrow$ 过 $\nu\left[\nu<\varkappa_{n}\right.$ and $A$ is many-one reducible to $\left.K_{n}(v)\right]$.

Thus if $\pi_{n}(v)=\left\{A \mid A\right.$ is many-one reducible to $\left.K_{n}(v)\right\}$, the classes $\pi_{n}(v)$ for $\nu<\varkappa_{n}$ form a hierarchy on the sets recursive in $r_{n}^{\#}$, that is on $\mathcal{R}_{n+1} \frown$ $\cap c \mathcal{R}_{n+1}$.

We do not know whether or not these are proper hierarchies - that is whether

$$
\mu<\nu<\varkappa_{L} \rightarrow \pi_{n}(\mu) \neq \pi_{n}(\nu),
$$

although we conjecture that they are. In any case we can of course make them proper by removing any superfluous classes. We wish to show that the resulting proper hierarchy still has order-type $\varkappa_{n}$. Let

$$
\begin{aligned}
K_{n}^{*}=\left\{u | u \in K _ { n } \text { and } \nabla v \left[|v|_{n}<|u|_{n} \rightarrow K_{n}\left(|u|_{n}\right)\right.\right. \text { is not many-one } \\
\text { reducible to } \left.\left.K_{n}\left(|v|_{n}\right)\right] \text { and } \nabla v\left(|v|_{n}=|u|_{n} \rightarrow u \leqslant v\right)\right\} .
\end{aligned}
$$

It is not hard to see that $K_{n}^{*}$ is recursively enumerable but not recursive in $r_{n}^{\#}$ and provides a unique notation for each $v<x_{n}$ such that $\pi_{n}(v)$ is strictly larger than any $\varkappa_{n}(\mu)$ for $\mu<v$. The mapping $/\left.\right|_{n}$ induces a unique mapping ||$_{n}^{*}$ of $K_{n}^{*}$ onto some ordinal $x_{n}^{*} \leqslant x_{n}$. Suppose for a contradiction that $x_{n}^{*}<x_{n}$. Then there is a unique ordinal-preserving function $\varphi$ mapping $K_{n}\left(x_{n}^{*}\right)$ onto $K_{n}^{*}$. In fact, $\varphi$ can be defined by:

$\varphi(u) \simeq v \leftrightarrow u \in K_{n}\left(x_{n}^{*}\right)$ and $v \in K_{n}^{*}$ and $\nabla \alpha[$ if $\alpha$ is an ordinal-preserving map of $K_{n}(|u|)$ onto $K_{n}^{*}\left(|v|_{n}^{*}\right)$, then $\left.\alpha(u)=v\right]$.
The function quantifier occurring in the definition is really only a Suslin quantifier $(\nabla \alpha$ 正 $p-\bar{\alpha}(p)-)$ so a bit of computation using a uniform version of (12) and its analogue for $K_{n}^{*}$ shows that $\varphi$ is partial recursive in $r_{n}^{\#}$. Then since for any $v$,

$$
v \in K_{n}^{*} \leftrightarrow \mathbb{H} u\left[u \in K_{n}\left(x_{n}^{*}\right) \text { and } \varphi(u) \simeq v\right]
$$

and $K_{n}\left(x_{n}^{*}\right)$ is the domain of $\varphi, K_{n}^{*}$ is recursive in $r_{n}^{\#}$, which is a contradiction. Thus we have proved

THEOREM 4. For $u \in K_{n}^{*}$, let $\varkappa_{n}^{\prime}\left(|u|_{n}^{*}\right)=\varkappa_{n}\left(|u|_{n}\right)$. Then the classes $\varkappa_{n}^{\prime}(v)$ form a proper hierarchy of length $\varkappa_{n}$ which exhausts $R_{n+1} \cap a R_{n+1}$.

We note that $\varkappa_{n}$ is the same as the ordinal $\omega_{R_{n}}$ of [Am 2]. The hierarchy constructed there also exhausts $R_{n+1} \cap a R_{n+1}$ (see [Hi 2]).

4. Inductive definability. A function $\Phi: \mathfrak{T}(N) \rightarrow \mathfrak{T}(N)$ (here called an (inductive) operator) is monotone iff $A \subseteq B$ implies $\Phi(A) \subseteq \Phi(B)$. Any monotone operator has a smallest fixed point

$$
\Phi^{*}=\bigcap\{A \mid \Phi(A) \subseteq A\}
$$

If $\mathrm{C}$ is a class of operators, a set $A$ is $\mathrm{C}$-monotone inductively definable iff $A$ is many-one reducible to $\Phi^{*}$ for some monotone operator $\Phi$ in $\mathrm{C}$.

For any partial function $\theta$, let $Z_{\theta}=\{m \mid \theta(m) \simeq 0\}$. An operator is in $\Sigma_{n}^{r}, \ldots$ iff the relation $m \in \Phi\left(Z_{a}\right)$ is a $\Sigma_{n}^{r}, \ldots$ subset of $N \times N^{N}$. In this section we present a generalization of the following two results of [Kl] and [Ac], Theorem 1, 2, respectively: for all $A \subseteq N$,

$$
A \in c \Re_{1}\left(=\Pi_{1}^{1}\right) \leftrightarrow A \text { is } \Pi_{1}^{0} \text {-monotone inductively definable, }
$$

$A \in C R_{2}\left(=\right.$ r.e. in $\left.E_{1}^{\#}\right) \leftrightarrow A$ is $\Sigma_{1}^{1}$-monotone inductively definable.

DEFINITION. For any inductive operator $\Phi$ and any $n$ :

(a) $\Phi$ is $R_{n} \leftrightarrow$ for some partial recursive functional $g$ and all $\theta$,

$$
\Phi\left(Z_{\theta}\right)=\left\{m \mid \mathbb{H} \beta_{\beta \in M_{n}} \nabla q[g(\theta, m, \beta(q)) \simeq 0]\right\},
$$

(b) $\Phi$ is weakly $R_{n} \leftrightarrow$ for some recursive functional $g$ and all $\alpha$,

$$
\Phi\left(Z_{\alpha}\right)=\left\{m \mid \text { 过 } \beta_{\beta \in M_{n}} \nabla q[g(\alpha, m, \beta(q))=0]\right\} \text {. }
$$

It is immediate that $\Phi$ is weakly $R_{0}\left(R_{1}\right)$ just in case $\Phi$ is $\Pi_{1}^{0}\left(\Sigma_{1}^{1}\right)$, so from (14) we have for all $A \subseteq N$ :

(15) $\quad(n=0,1) A \in c \mathcal{R}_{n+1} \leftrightarrow A$ is weakly $R_{n}$-monotone inductively definable.

We do not know whether or not (15) holds for all $n$. 
THeOrem 5. For all $n$ and all $A \subseteq N$ :

$A \in c \mathcal{R}_{n+1} \leftrightarrow A$ is $R_{n}$-monotone inductively definable .

Proof. Suppose first that $A$ is many-one reducible to $\Phi^{*}$ for some $R_{n}$ operator $\Phi$. Note that $\Phi$ is automatically monotone. Let $g$ be as in the definition and set

so that

$$
f(\theta, m) \simeq r_{n}^{\#}(\lambda p \cdot g(\theta, m, p)),
$$

$$
m \in \Phi\left(Z_{\theta}\right) \leftrightarrow f(\theta, m) \simeq 0 .
$$

Clearly $f$ is partial recursive in $r_{n}^{*}$, so by the first recursion theorem there is a function $\theta^{*}$ partial recursive in $r_{n}^{*}$ such that

$$
\theta^{*}(m) \simeq f\left(\theta^{*}, m\right),
$$

and $\theta^{*}$ is the smallest such function. Hence

$$
\theta^{*}(m) \simeq 0 \leftrightarrow m \in \Phi^{*},
$$

so $\Phi^{*}$ and $A$ are recursively enumerable in $r_{n}^{*}$ and thus in $c \mathcal{R}_{n+1}$ by Corollary. B.

For the other direction, suppose $A \epsilon c \mathcal{R}_{n+1}$ and let $F$ be a recursive family such that $A=N \sim R_{n+1}(F)$, that is

$$
m \in A \leftrightarrow \nabla \alpha_{\alpha \in M_{n+1}} \mathbb{H} p[m \notin F(\alpha(p))] .
$$

Let $\Phi$ be the monotone operator defined by:

$$
\langle m, s\rangle \epsilon \Phi(B) \leftrightarrow m \notin F(s) \text { or } \mathbb{H} \beta_{\beta \in M_{n}} \nabla q[\langle m, s\langle\beta(q)\rangle\rangle \epsilon B] .
$$

Then $\Phi$ is an $R_{n}$ operator since

$$
\langle m, s\rangle \in \Phi\left(Z_{\theta}\right) \leftrightarrow \mathbb{H} \beta_{\beta \in M_{n}} \forall q\left[m \notin F^{\prime}(s) \text { or } \theta(\langle m, s\langle\beta(q)\rangle\rangle) \simeq 0 \mid .\right.
$$

We will show

$$
\langle m, s\rangle \epsilon \Phi^{*} \leftrightarrow \nabla \alpha_{a \in M_{n+1}} \mathbb{H} p[m \notin F(s * \alpha(p))],
$$

from which follows

$$
m \in A \leftrightarrow\langle m, \varnothing\rangle \epsilon \Phi^{*} .
$$

First suppose $\langle m, s\rangle \notin \Phi^{*}$ and let $\alpha_{0}$ enumerate the set of all $t$ such that $\forall u\left(u \subseteq t \rightarrow\langle m, s * u\rangle \notin \Phi^{*}\right)$. We claim $a_{\mathrm{c}} \in M_{n+1}=\boldsymbol{R} M_{n}^{0}$. Clauses (i) and (ii) of (1) are obviously satisfied. Furthermore, for any $t$,

$$
\begin{aligned}
\langle m, s * t\rangle \notin \Phi^{*} & \rightarrow\langle m, s * t\rangle \notin \Phi\left(\Phi^{*}\right) \\
& \rightarrow \forall \beta_{\beta \in M M_{n}} \mathbb{H} q\left[\langle m, s * t\langle\beta(q)\rangle\rangle \notin \Phi^{*}\right] \cdot \\
& \rightarrow \mathbb{H} \gamma_{\gamma \in M M_{n}^{0}} \nabla r\left[\langle m, s * t\langle\gamma(r)\rangle\rangle \notin \Phi^{*}\right],
\end{aligned}
$$

and thus (iii) is also satisfied. By definition,

$$
\nabla p\left[\left\langle m, s * a_{0}(p)\right\rangle \notin \Phi^{*}\right],
$$

so, in particular,

$$
\nabla p\left[m \notin F\left(s * a_{\mathrm{n}}(p)\right)\right],
$$

which yields the negation of the right side of (16).

For the left-to-right implication of (16), let

$$
C=\left\{\langle m, s\rangle \mid \nabla \alpha_{\alpha \in M_{n+1}}\{p[m \notin F(s * \alpha(p))]\}\right. \text {. }
$$

By the definition of $\Phi^{*}$ it will suffice to show $\Phi(C) \subseteq C$. Suppose $\langle m, s\rangle$ $\epsilon \Phi(C)$. If $m \notin F(s)$, then $m \notin F(s * \emptyset)$ so $\langle m, s\rangle \in C$ by property (i) of (1). Otherwise, for some $\beta_{0} \in M_{n}$,

$$
\nabla q\left[\left\langle m, s\left\langle\beta_{0}(q)\right\rangle\right\rangle \epsilon C\right] .
$$

For any $\alpha \in M_{n+1}$, it follows from (i) and (iii) of (1) and the definition of $M_{n}^{0}$ that there exists $q_{0}$ such that $\left\langle\beta_{0}\left(q_{0}\right)\right\rangle \in \operatorname{Rg} \alpha$. Let $\gamma$ enumerate the set of $t$ such that $\left\langle\beta_{0}\left(q_{0}\right)\right\rangle t \in \operatorname{Rg} \alpha$. It is easily checked that $\gamma \in M_{n+1}$, so by the definition of $C$,

and thus

$$
\text { 但 } r\left[m \notin F\left(s\left\langle\beta_{0}\left(q_{0}\right)\right\rangle * \gamma(r)\right)\right],
$$

$$
\mathbb{A} p[m \notin F(s * \alpha(p))] \text {. }
$$

Since $\alpha$ was an arbitrary member of $M_{n+1}$, this shows $\langle m, s\rangle \epsilon C$ and completes the proof.

We conclude by mentioning some open questions concerning the relationships which hold among $R_{n}$ and weakly $R_{n}$ inductive operators. We know only the following:

LEMNA. For any monotone operator $\Phi$ and any $n$ :

(a) $\Phi$ is $R_{n}$ and $n>0 \rightarrow \Phi$ is weakly $R_{n}$,

(b) $\Phi$ is weakly $R_{0} \rightarrow \Phi$ is $R_{1}^{0}$,

(c) $\Phi$ is weakly $R_{1} \rightarrow \Phi$ is $R_{1}$.

Proof. (a) Extending a partial recursive $g$ to one that is total on total arguments requires only a number quantifier. For $n>0$, this can be "absorbed" into the prefix $\mathbb{H} \alpha_{a \in M_{n}} \nabla p$.

(b) For any monotone $\Phi$,

$$
m \in \Phi\left(Z_{\theta}\right) \leftrightarrow \nabla \alpha\left[Z_{\theta} \subseteq Z_{\alpha} \rightarrow m \in \Phi\left(Z_{\alpha}\right)\right] .
$$

If $\Phi$ is weakly $R_{0}$, this is easily put into $R_{1}^{0}$ form.

(c) is proved similarly using the fact that for monotone $\Phi$ 
We do not know whether either (b) or (c) can be extended to larger $n-$ that is whether for any $n>1$ and all monotone $\Phi$,

$$
\Phi \text { is weakly } R_{n} \rightarrow \Phi \text { is } R_{n+1}^{0} \text {, or }
$$

$\Phi$ is weakly $R_{n} \rightarrow \Phi$ is $R_{n}$.

Either would suffice to extend (15) to such $n$, since either would imply that if $\Phi$ is weakly $R_{n}$, the $f$ defined from $\Phi$ in the proof of Theorem 5 is partial recursive in $r_{n}^{*}$.

\section{References}

[Ac] P. Aczel, Representability in some systems of second order arithmetic, Israel J. Math. 8 (1970), pp. 309-328.

[Am 1] V. I. Amstislavskĭ, Effective $R$-sets and transfinite extensions of recursive hierarchies (Russian), Fund. Math. 68 (1970), pp. 61-86. English summary in Soviet Math. Dokl. 9 (1968), pp. 703-706.

[Am 2] - On the decomposition of a field of sets obtained by an $R$-operation over recursive sets (Russian), Dokl. Akad. Nauk SSSR 191 (1970), pp. 743-746. English translation in Soviet Math. Dokl. 11 (1970), pp. 419-422.

[En] H. B. Enderton, Hierarchies in recursive function theory, Trans. Amer. Math. Soc. 111 (1964), pp. 457-471.

[Hi 1] P. G. Hinman, Hierarohies of effective desoriptive set theory, Trans. Amer. Math. Soc, 142 (1969), pp. 111-140.

[Hi 2] Review of [Am 2], Jour. Symb. Log. 37 (1972), pp. 409-410.

[Kl] S. C. Kleene, On the forms of predicates in the theory of constructive ordinals (second paper), Amer. Jour. Math. 77 (1955), pp. 405-428.

[Kr-Ro] D. Kreider and H. Rogers, Jr., Constructive versions of ordinal number classes, Trans. Amer. Math. Soc. 100 (1961), pp. 325-369.

UNIVERSITY OF MICHIGAN

Ann Arbor, Michigan

Reģu par la Rédaction le 10. 5. 1971

\section{Ordres "C.A.C." \\ par}

\section{B. Leclerc et B. Monjardet (Paris)}

Résumé. Les ordres "C.A.C." (chain-antichain complete) ont été définis par Grillet dans [5]; ce sont les ordres où toute partie libre maximale rencontre toute chaine maximale; on peut aussi énoncer cette propriété en termes d'hypergraphe ([2]): l'hypergraphe défini par les chaines maximales d'un ordre "C.A.C." appartient à la classe des hypergraphes dont l'ensemble des transversales fortement stables égale l'ensemble des parties fortement stables maximales. Ici, nous développons l'étude des ordres "C.A.C.", dans le cas fini.

Au premier paragraphe, nous en donnons une caractérisation du type Kuratowski (théorème 1). Au paragraphe 2, nous caractérisons les ordres gradués C.A.C. et nous donnons quelques résultats sur leur dimension. Au troisième paragraphe, nous étudions les treillis C.A.C., nous caractérisons ces treillis et les treillis gradués C.A.C.; nous donnons plusieurs caractérisations des treillis modulaires C.A.C. (théorème 2); on déduit immédiatement de ces résultats que ces ordres sont de dimension deux.

0. Définitions et notations. Nous considérons un ensemble $E$ ordonné; la relation d'ordre sur $E$ sera notée $O$ ou $\leqslant$; nous noterons $x O y$ ou $x \leqslant y$; la relation de couverture associée à l'ordre sera notée $\zeta: x \zeta y$ s'il n'existe pas $z$ tel que $x<y<z$; la relation d'incomparabilité associée d̀ l'ordre sera notée $I: x I y$ si $x \nless y$ et $y \nless x ; x$ est comparable à $y$ si $x \leqslant y$ ou $y \leqslant x$.

Une partie libre $L$ de l'ensemble ordonné $(E, \leqslant)$ est une partie de $E$ dont les éléments sont deux à deux incomparables (on dit aussi partie indépendante, sous-ensemble stable intérieurement, antichaine); une chaine de l'ensemble ordonné est une partie de $E$ dont les éléments sont deux à deux comparables (c'est à dire un sous-ensemble totalement ordonné). Nous notons $\mathcal{L}$ l'ensemble des parties libres, $\mathfrak{L}^{m}$ l'ensemble des parties libres maximales; nous notons $\&$ l'ensemble des chaines, $\varepsilon^{m}$ l'ensemble des chaines maximales.

La dimension (au sens de Dushnik-Miller) d'un ordre $O$ est le nombre minimum d'ordres totaux dont $O$ est intersection; nous la notons $\operatorname{dim}(O)$ ([4] ou [1] chapitre VI)

Un hypergraphe $($ simple $) H=(E, \mathcal{A})$ est le couple formé d'un ensemble fini $E$ et d'un ensemble de parties non vides de $E$ appelées arêtes: $\mathcal{A}=\left\{A_{i}, i \in I\right\} ;$ une transversale de $H$. est une partie de $E$ rencontrant 